\title{
Adaptive Digital Correction of Analog Errors in MASH ADC's-Part I: Off-Line and Blind On-Line Calibration
}

\author{
Gert Cauwenberghs, Member, IEEE and Gabor C. Temes, Life Fellow, IEEE
}

\begin{abstract}
Cascaded delta-sigma (MASH) modulators for higher order oversampled analog-to-digital conversion rely on precise matching of contributions from different quantizers to cancel lower order quantization noise from intermediate delta-sigma stages. This first part of the paper studies the effect of analog imperfections in the implementation, such as finite gain of the amplifiers and capacitor ratio mismatch, and presents algorithms and architectures for digital correction of such analog imperfections, as well as gain and spectral distortion in the signal transfer function. Digital correction is implemented by linear finite-impulse response (FIR) filters, of which the coefficients are determined through adaptive off-line or on-line calibration. Of particular interest is an on-line "blind" calibration technique, that uses no reference and operates directly on the digital output during conversion, with the only requirement on the unknown input signal that its spectrum be bandlimited. Behavioral simulations on dual-quantization oversampled converters demonstrate near-perfect adaptive correction and significant improvements in signal-to-quantization-noise performance over the uncalibrated case, using as few as 5 FIR coefficients. An alternative on-line adaptation technique using test signal injection and experimental results from silicon are presented in the second part, in a companion paper [1].
\end{abstract}

Index Terms-Adaptation, analog-to-digital conversion, blind equalization, delta sigma, digital correction, MASH, mismatch, sigma delta.

\section{INTRODUCTION}

$\mathbf{M}$ ASH-LIKE cascaded structures of delta-sigma modulators [2] provide high-order noise shaping through combining the differentiated digital outputs from the multiple stages in a way to cancel out the lower order quantization error contributions from all intermediate stages [3], [4]. However, such structures are very sensitive to the analog accuracy of implementation, typically limiting the resolution of analog-to-digital (A/D) conversion to less than 14 bits, regardless of the order of the noise shaping. Likewise, the

Manuscript received November 1999; revised March 2000. The work of G Cauwenberghs was supported in by the National Science Foundation (NSF) under NSF Career Award MIP-9702346, by Northrop Grumman (Maryland Industrial Partnership), and by Lucent Technology. The work of G. Temes was supported by the NSF Center for Design of Analog-Digital Integrated Circuits (CDADIC). This paper was recommended by Associate Editor T. Fiez.

G. Cauwenberghs is with the Department of Electrical and Computer Engineering, Johns Hopkins University, Baltimore, MD 21218 USA.

G. C. Temes is with the Department of Electrical and Computer Engineering, Oregon State University, Corvallis, OR 97331 USA.

Publisher Item Identifier S 1057-7130(00)05808-0. performance of the Leslie-Singh modulator [5], [6] depends critically on precise cancellation of error terms originating from two quantizers of different resolution. The problem of sensitivity to the precision of analog implementation arises generally in modulators which contain more than one quantizer in the signal path.

The analog sensitivity can be reduced, to a limited extent, through careful design of the modulator architecture [7], [8]. The alternative to high-precision analog design, which we describe here, is to correct for analog imprecision in the digital domain, trading a small increase in the implementation complexity of the digital part for a significant increase in effective analog precision. Digital correction of dual-quantization and cascaded delta-sigma modulators has been investigated, using off-line [9] and on-line [10]-[13] adaptive methods, and recently demonstrated in analog hardware [14].

We show that typical analog sources of error in multiple quantization modulators, such as MASH and Leslie-Singh structures, can be virtually eliminated through simple linear digital correction using finite-impulse response (FIR) digital filters, of which the coefficients are determined through adaptive calibration. In this first part of this paper, the approach taken to adaptive digital correction is in essence a calibration procedure, either using a well-defined (pseudo-random) two-level reference signal sequence applied to the analog input of the modulator, or by appropriately bandlimiting the input signal so that the signal is zero (and thus, equally well-defined) over a dedicated "calibration band" outside of the signal band. Both techniques apply to general multistage structures of cascaded delta-sigma modulators, with multibit quantization in the last stage [2] or an additional multibit quantizer as output stage [5]. The second part sequel to this paper [1] describes another adaptive method, where test signals are injected at critical points of the architecture and their contributions auto-zeroed at the output.

Section II covers sources of analog imprecision, and a corresponding linear model for analog parts of the modulator. Digital FIR correction of these linear errors is described in Section III, quantifying the effect of analog imprecision on MASH performance, both with and without digital correction. Off-line and on-line calibration techniques are presented and demonstrated with behavioral simulation examples in Section IV. Finally, we conclude in Section VI which also opens the subject of the companion paper [1]. 


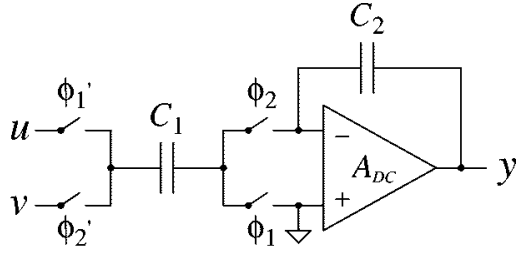

(a)

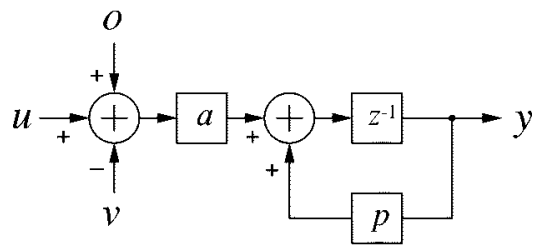

(b)

Fig. 1. Typical single-ended switched-capacitor realization of (a) an integrator and (b) corresponding linear analog model.

\section{ANALOG MODELING}

\section{A. Sources of Analog Imprecision}

The input integrator in the analog implementation of a delta-sigma modulator (performing both "delta" subtraction and "sigma" accumulation) comprises the most critical part, since it is directly at the input terminal in the signal path. A typical single-ended implementation using switched-capacitor circuits [15] is shown in Fig. 1(a). Ideally, the circuit implements the function

$$
y[n+1]=y[n]+a(u[n]-v[n])
$$

with gain $a=C_{1} / C_{2}$ controlled by the design geometry of capacitors $C_{1}$ and $C_{2}$. In practice, (1) is affected by the following factors.

1) Capacitor mismatch in $C_{1}$ and $C_{2}$, affecting the gain $a$ directly.

2) Finite amplifier gain $A_{D C}$, affecting both the gain $a$ and a leakage factor $p$ in the integration

$$
y[n+1]=p y[n]+a(u[n]-v[n])
$$

with

$$
\begin{aligned}
& a=\frac{\frac{C_{1}}{C_{2}}}{1+\frac{1}{A_{D C}}\left(1+\frac{C_{1}}{C_{2}}\right)} \\
& p=\frac{1+\frac{1}{A_{D C}}}{1+\frac{1}{A_{D C}}\left(1+\frac{C_{1}}{C_{2}}\right)} .
\end{aligned}
$$

3) Amplifier settling time, further affecting both integrator gain $a$ and leakage $p$.

4) Amplifier offset and (thermal and 1/f) noise, which can be referred to the input in the form of an additive offset $o$.

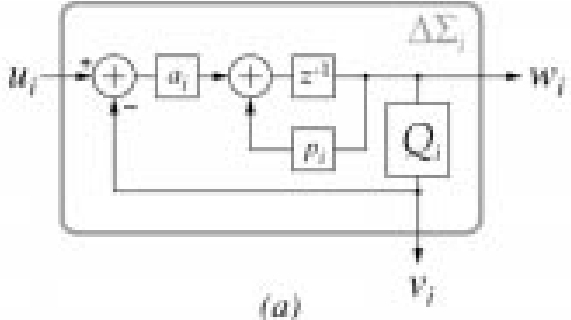

(a)

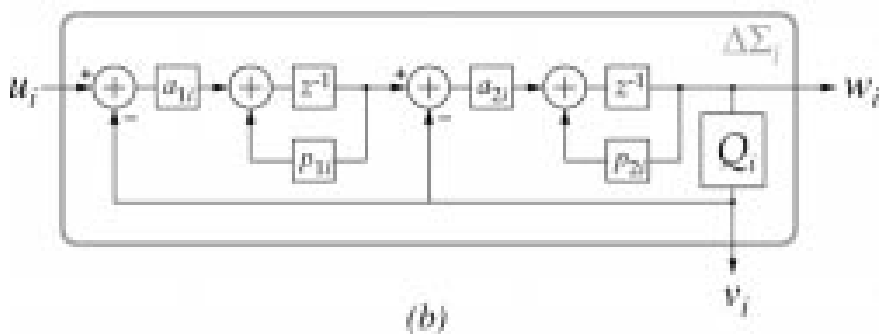

Fig. 2. Delta-sigma modulator models accounting for analog imperfections: (a) first-order modulator and (b) second-order modulator.

Correlated double sampling (CDS) or other offset compensation circuit techniques allow to cancel the DC component of this term so that it may safely be ignored.

5) Nonlinearity in the components (amplifier, capacitors, switch injection noise, etc.).

Nonlinearities are detrimental to the signal, and cannot easily be digitally compensated for. On the other hand, linear errors can be accounted for in the digital domain using the analog model of Fig. 1(b), with unknown and possibly variable values $a_{i}$ and $p_{i}$ for the $i$ th integrator in the modulator.

\section{B. Linear Error Model of Modulator Structures}

Typical structures used as first and second order delta-sigma modulators are shown in Fig. 2(a) and (b). Besides modeling capacitive mismatch $C_{1} / C_{2}$, the gain parameters $a_{i}$ may serve the purpose of improving the stability of noise shaping and increasing the input dynamic range in the standard second-order modulator [16] with single-bit feedback, for a nominal gain $a$ less than one ( $a=0.5)$ [17]. From (2) in the $z$-domain, either modulator satisfies the linear FIR recurrence relation

$$
G_{i}(z) V_{i}(z)=z^{-l_{i}} U_{i}(z)-H_{i}(z) W_{i}(z)
$$

or, equivalently, in terms of the quantization error (residue) $E_{i}=V_{i}-W_{i}$

$$
\left(G_{i}(z)+H_{i}(z)\right) V_{i}(z)=z^{-l_{i}} U_{i}(z)+H_{i}(z) E_{i}(z)
$$

where, for the first-order modulator $\left(l_{i}=1\right)$

$$
G_{i}(z)=z^{-1}, \quad H_{i}(z)=\frac{1-p_{i} z^{-1}}{a_{i}}
$$

and for the second-order modulator $\left(l_{i}=2\right)$

$$
\begin{aligned}
& G_{i}(z)=z^{-2}+z^{-1} \frac{1-p_{1 i} z^{-1}}{a_{1 i}} \\
& H_{i}(z)=\frac{1-p_{1 i} z^{-1}}{a_{1 i}} \frac{1-p_{2 i} z^{-1}}{a_{2 i}} .
\end{aligned}
$$




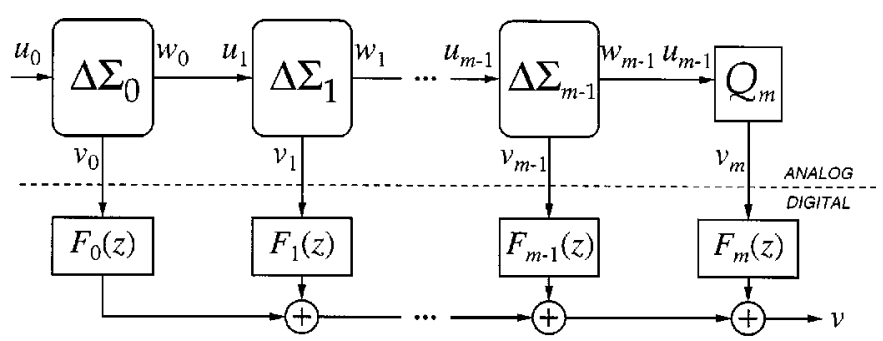

(a)

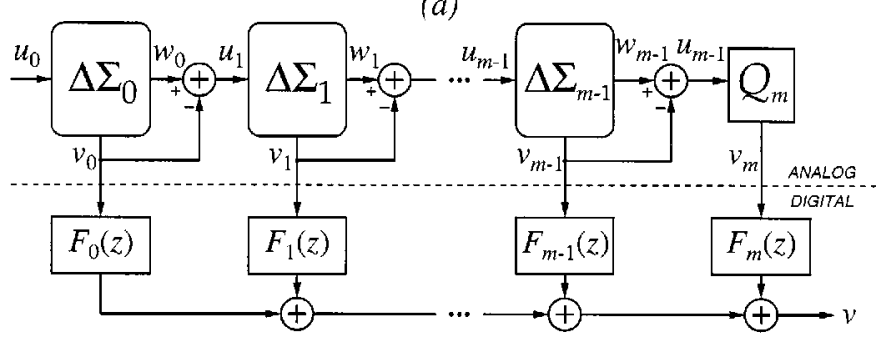

(b)

Fig. 3. Cascaded delta-sigma modulator topologies: MASH modulator with (a) integrator coupled outputs and (b) residue coupled outputs.

\section{Multistage (MASH) Delta-Sigma Modulation}

A MASH modulator is obtained by cascading individual delta-sigma modulators $\Delta \Sigma_{i}$ from analog inputs $U_{i}(z)$ to analog outputs $W_{i}(z)$ (or residues $E_{i}(z)$ ), and combining the digital outputs $V_{i}(z)$ accordingly. An optional final stage with multibit quantizer can be used to further boost the signal-to-quantization-noise ratio (SQNR).

\section{A. Analog Modulator}

Two possible scenarios of interest are depicted in Fig. 3. In the first, the integrator output (prior to quantization) is fed directly to the input of the next stage: $W_{i}(z) \equiv U_{i+1}$ for $i=0 \cdots, m-$ 1. From (4) and Fig. 3(a)

$$
\begin{aligned}
G_{i}(z) V_{i}(z) & =z^{-l_{i}} U_{i}(z)-H_{i}(z) U_{i+1}(z), \quad i=0 \cdots, m-1 \\
V_{m}(z) & =U_{m}(z)+E_{m}(z) .
\end{aligned}
$$

In the second scenario, shown in Fig. 3(b), only the quantization residue $-E_{i}(z)=W_{i}(z)-V_{i}(z)$ is fed to the next stage. The advantage of this scheme is an improvement of up to $6 \mathrm{~dB}$ in dynamic range (a factor of two in the residue, assuming 1-bit quantization), at the expense of a slight complication in the analog design (to construct the residue). Also, the residue subtraction scheme minimizes distortion of the signal due to nonlinearities in subsequent stages [18]. Results of digital linear correction are identical for both schemes (through a trivial transformation), and we proceed with the one in Fig. 3(a).

\section{B. Digital Correction}

An ideal cascaded delta-sigma modulator cancels the quantization noise from intermediate delta-sigma stages through matched FIR filtering of the quantized outputs [2], [3], [5]. By the same token, a corrected digital output $V(z)$ can be obtained, for a modulator with analog imperfections, by combining the quantized outputs $V_{i}(z)$ through digital (FIR) filters $F_{i}(z)$

$$
V(z)=\sum_{i=0}^{m} F_{i}(z) V_{i}(z)
$$

which, according to (8), translates into a combination of signal and quantization noise

$$
\begin{aligned}
V(z)=\operatorname{STF}(z) U_{0}(z)+\sum_{i=0}^{m-1} \mathrm{NTF}_{i}(z) & U_{i+1}(z) \\
& +\mathrm{NTF}_{m}(z) E_{m}(z)
\end{aligned}
$$

with signal transfer function

$$
\operatorname{STF}(z)=z^{-l_{0}} \frac{F_{0}(z)}{G_{0}(z)}
$$

and noise transfer functions

$$
\begin{aligned}
\mathrm{NTF}_{i-1}(z) & =z^{-l_{i}} \frac{F_{i}(z)}{G_{i}(z)}-H_{i-1}(z) \frac{F_{i-1}(z)}{G_{i-1}(z)} \\
& i=1 \cdots, m-1 \\
\mathrm{NTF}_{m-1}(z) & =F_{m}(z)-H_{m-1}(z) \frac{F_{m-1}(z)}{G_{m-1}(z)} \\
\mathrm{NTF}_{m}(z) & =F_{m}(z) .
\end{aligned}
$$

An appropriate choice for the coefficients of the FIR filters $F_{i}$ is one that cancels all noise transfer functions, except the very last. Ideal digital correction

$$
\begin{aligned}
F_{i}^{c}(z) & =z^{-\sum_{k=i+1}^{m-1} l_{k}} G_{i}(z) \prod_{k=0}^{i-1} H_{k}(z), \\
i & =1 \cdots, m-1
\end{aligned}
$$

equalizes the signal transfer function and produces noise shaping of cumulative order $L=\sum_{k=0}^{m-1} l_{k}$

$$
\begin{aligned}
V^{c}(z) & =\operatorname{STF}^{c}(z) U_{0}(z)+\mathrm{NTF}_{m}^{c}(z) E_{m}(z) \\
& =z^{-L} U_{0}(z)+\prod_{k=0}^{m-1} H_{k}(z) E_{m}(z) \\
& \approx z^{-L} U_{0}(z)+\left(\frac{1-p z^{-1}}{a}\right)^{L} E_{m}(z)
\end{aligned}
$$

where $a$ and $p$ denote the nominal gains and pole errors of the integrators in the cascade. In other words, the noise-shaping (SQNR) performance of the digitally corrected cascade structure is equivalent to that of a (hypothetically stable) single-stage design of the same order $L$, same number of bits in the quantizer $Q_{m}$, and with identical gain $A_{D C}$ of the amplifiers. The performance loss due to the persistence of the pole errors in $\mathrm{NTF}_{m}$ 


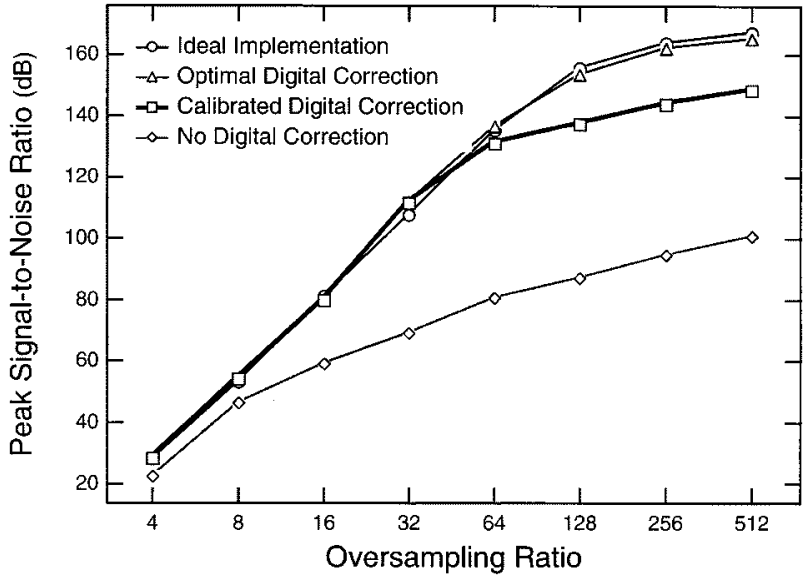

Fig. 4. Simulated peak signal-to-noise performance of the oversampled converter for different oversampling ratios, with digital correction of analog implementation errors. Gain errors $\left(\left|a_{i j}-a\right|\right)$ are nominally $5 \%$, and pole errors $\left(1-p_{i j}\right)$ are nominally $1 \%$.

is, for all practical purposes, negligible for reasonable values of amplifier gain $A_{D C}$ [2].

\section{AdAPTIVE CALIBRATION}

\section{A. Off-Line Reference-Based Calibration}

The key to the practical application of digital correction is a method to determine the values of the coefficients in the $F_{i}$ filters from physical observations, with no prior knowledge about the $G_{i}$ and $H_{i}$. One possibility is to state this task as a regression problem, and derive values for the coefficients from a least-squares error criterion on the digital output sequence under application of a well-defined analog input sequence [9]. In essence, the technique corresponds to a calibration of the signal transfer function of the oversampled converter, in combination with a maximum reduction of the in-band quantization noise.

Choosing a random-sign constant-amplitude analog input sequence $u^{\text {cal }}[n]$ to perform the calibration is most practical, since it avoids analog implementation errors while still covering most of the spectrum. Targeting (14), a least-squares error criterion on the input and output sequences is to minimize

$$
E\left(F_{0}, \cdots F_{m}\right)=\sum_{n=1}^{N}\left(\mathcal{L}_{c} v[n]-\mathcal{L}_{c} u^{\mathrm{cal}}[n-L]\right)^{2}
$$

where $N$ is the calibration sample size, and the linear time-invariant operator $\mathcal{L}_{c}(\cdot)$ represents the decimation low-pass filter. To be effective, the low-pass filter $\mathcal{L}_{c}$ needs to cut off near the signal Nyquist frequency, and needs to roll off faster than the noise spectrum rises (e.g., $6(L+1) \mathrm{dB} / \mathrm{oct}$ ). From (9), the minimization of (15) becomes a standard linear regression procedure, with the FIR coefficients of $F_{i}$ as parameters, $\mathcal{L}_{c} u^{\text {cal }}[n-$ $L]$ as the dependent variable, and the $\mathcal{L}_{c} v_{i}[n]$ plus their delayed values as independent variables. The solution is therefore unique, and can be obtained in practice with any standard linear regression method (recursive least squares, conjugate gradient, etc.).

As a numerical example, we consider the case $m=2$ in Fig. 3(b), with two cascaded second-order delta-sigma modu-

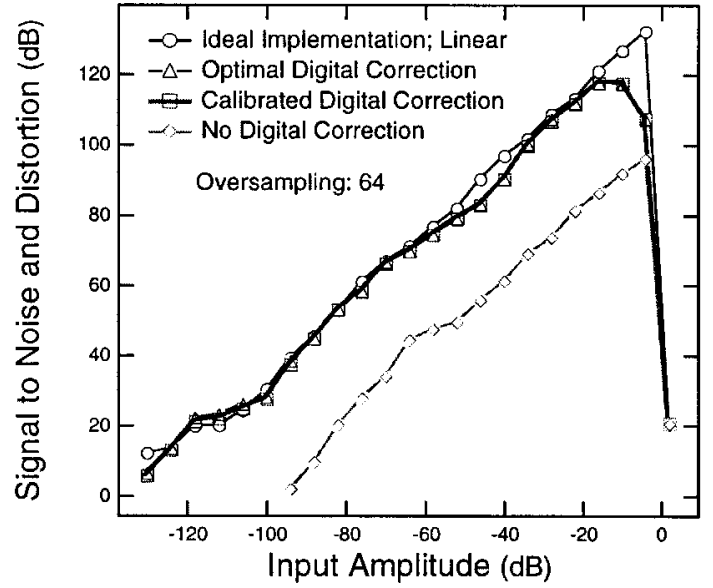

(a)

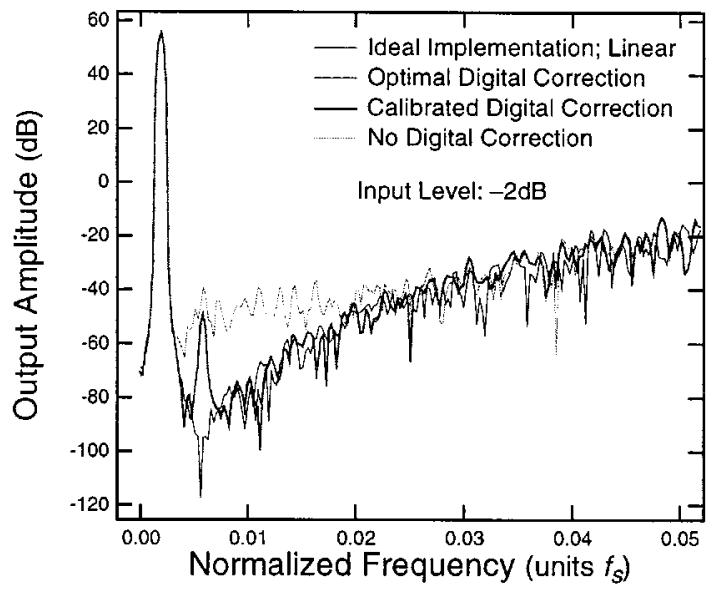

(b)

Fig. 5. Effect of nonlinear amplifier gain on noise and distortion performance of the oversampled converter, with digital correction of linear analog imperfections errors. (a) SNDR for different input levels, with 64 times oversampling. (b) Output amplitude spectrum for a $-2 \mathrm{~dB}$ full-scale sinusoidal input. Gain errors $\left(\left|a_{i j}-a\right|\right)$ are nominally $0.5 \%$, linear pole errors $\left(1-p_{i j}\right)$ are $0.1 \%$, and nonlinear pole errors are nominally $-5 \%$ relative to the linear error.

lators $\left(l_{0}=l_{1}=2\right)$, the first one with 1-bit quantizer, and the second with a 6-bit quantized output.

Fig. 4 shows simulation results of the effect of digital correction, using the above calibration with $N=1,024$, on the SNR performance of the oversampled converter. Also shown are the results of optimal digital correction according to (13), which are indistinguishable from those obtained for an ideal converter without analog imperfections. Fig. 4 demonstrates that the calibration method is effective for the compensation of analog imperfections for all practical SNR levels of interest (up to $130 \mathrm{~dB}$, corresponding to 21 ENOB's).

Since the presented digital correction scheme is linear, any quantization noise and harmonic distortion due to nonlinearities in the analog implementation cannot be eliminated from the output. An important source of nonlinearities are signal-dependent gain variations in the integrator amplifiers (nonlinear $p_{i j}$ ). The simulations in Fig. 5 indicate that the effect of reasonably small nonlinearities on performance is not drastic. The net effect 
is saturation of the signal-to-noise-and-distortion ratio (SNDR) near full-range amplitudes, shown in Fig. 5(a). Harmonic distortion is visible in the spectrum of the (optimally corrected) calibrated output in Fig. 5(b).

\section{B. Pseudo On-Line Interleaved Reference-Based Calibration}

The main disadvantage of the above calibration procedure is that it is off-line, interrupting the normal operation of the data converter by presenting the calibration pseudo-random sequence at the input. A direct on-line extension is obtained in a configuration containing at least two converters, where each one in turn is removed from the conversion process for off-line calibration, while the other(s) remain(s) active. Such arrangement allows calibration to proceed in the background, for continuous tracking of slow variations in the analog imperfections of the modulators. With two converters in one module, the cost in overhead to provide on-line operation is $100 \%$. However, for a linear array of $P$ converters operating in parallel, the relative overhead of one extra converter reduces to $1 / P$, asymptotically approaching zero for large $P$. In such arrangement, the converters could be calibrated in circular periodic fashion, each one covered once every $P+1$ calibration cycles. This arrangement is attractive for use in high-speed multiplexed [19], multirate [20], [21], and interleaved [22] arrays of parallel converters. Since offset and gain errors of the individual converters are virtually eliminated along with the lower order quantization noise of their modulators, effects of cyclic pattern noise and nonlinear distortion in a multiplexed arrangement are significantly reduced.

\section{On-Line Reference-Based Calibration}

An intrinsically on-line alternative, allowing the reference signal to co-exist with the input signal without multiplexing, is to additively combine the input $U_{0}$ with a broadband (pseudo-random) reference signal $U_{0}^{\text {cal }}$, and employ spread-spectrum techniques (matched filtering) to adaptively

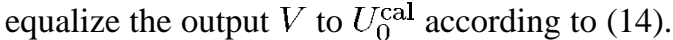

A particular on-line scheme is "test injection" [1], [12], [14], which additively injects reference signals $U_{i+1}^{\text {cal }}$ onto the $U_{i+1}$ nodes in between stages in Fig. 3, and adaptively cancels the corresponding $\mathrm{NTF}_{i}$ terms in $(10)(i=0, \cdots m-1)$ by auto-zeroing the contribution $U_{i+1}^{\text {cal }}$ to $V$ through matched filtering of $V$ with $U_{i+1}^{\mathrm{cal}}$. This technique is described in detail and experimentally demonstrated in Part II of the paper.

\section{Blind On-Line Calibration}

A reference-free alternative is a "blind" calibration technique that operates with nothing but the unknown input signal, and applies generally to pipelined algorithmic A/D converters, multistage oversampled A/D converters, and their combinations [13]. The technique assumes a strictly bandlimited input signal, sampled at a frequency $f_{s}$ greater than the Nyquist rate $f_{N}=2 f_{c}$, where $f_{c}$ is the signal bandwidth. The stopband $\left[f_{c}, f_{s}-f_{c}\right]$ is then reserved for calibration purposes, as illustrated schematically in Fig. 6. As with off-line calibration using a reference signal, the technique corrects for linear errors in the analog implementation by minimizing the quantization

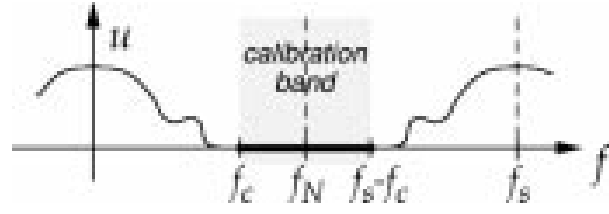

Fig. 6. Signal stopband for blind calibration.

error variance in the stopband through least-squares linear parameter estimation.

A fringe benefit of the oversampling is a significant frequency margin for calibration over the entire noise band $f_{N} / f_{s}=1 / R$, where $R$ is the oversampling ratio. The only complication arises from the frequency dependency of the quantization error in (14) through the noise shaping. The key is to match the noise-shaping of the quantization noise as faithfully as possible in the leastsquares formulation of the parameter estimates.

A high-pass filter $\mathcal{H}_{c}$ spanning the band $\left[f_{c}, f_{s}-f_{c}\right]$ removes the input signal $U_{0}$. In principle, an ideal high-pass filter is needed to completely eliminate the unknown signal for precise calibration. In practice, a filter with finite roll-off in the signal band is adequate as long as the signal leakage is below the quantization noise floor in the calibration band, which is fairly easy to accommodate with conventional filter structures. The quantization noise $E_{m}$ is modeled as white noise with uniform power spectrum over the calibration band. Thus, from (14) and (9), the coefficients of the FIR filters $F_{i}(z)$ are estimated by minimizing the variance of $E_{m}$ over the calibration band

$$
\begin{aligned}
\left|\mathcal{H}_{c}(z) E_{m}(z)\right|^{2} & =\left|\mathrm{NTF}_{m}^{-1}(z) \mathcal{H}_{c}(z) V(z)\right|^{2} \\
& \approx\left|\sum_{i=0}^{m} F_{i}(z) \mathcal{N}_{c}(z) V_{i}(z)\right|^{2}
\end{aligned}
$$

where $z \equiv e^{j \Omega}$ with $\Omega=2 \pi f / f_{s}$. The digital emphasis filter

$$
\mathcal{N}_{c}(z)=\left(\frac{a z^{-1}}{1-z^{-1}}\right)^{L} \mathcal{H}_{c}(z) \approx z^{-L} \mathrm{NTF}_{m}^{-1}(z) \mathcal{H}_{c}(z)
$$

serves to equalize the noise-shaping of the spectrum of $E_{m}$ in the estimation, besides removing the signal band of $U_{0}$. The error made in the approximation of the unknown noise-shaping in (17) does not affect the accuracy of the results to first order.

The least-squares formulation 16 is rank deficient, and one of the coefficients needs to be fixed in the optimization for a unique solution to exist. This is not surprising, since the nature of blind calibration leaves the signal gain undefined, in the absence of an absolute reference.

It is relatively straightforward to extend the blind calibration procedure to include adaptive calibration of linear errors in the quantizer $Q_{m}$, such as for an algorithmic (pipelined) A/D converter [13]. Using a linear model of the corrected output as a function of the A/D converted bits, the unknown coefficients are estimated jointly with the $F_{i}$ coefficients in the optimization (16). The same strategy could also be used to extend the on-line adaptive calibration to digital correction of analog implementation errors in a D/A converter providing multibit feedback in a $\Delta \Sigma$ integration loop, using a similar linear model of the corrected output in terms of the D/A converted bits. This is important, since multibit feedback significantly enhances the stability 


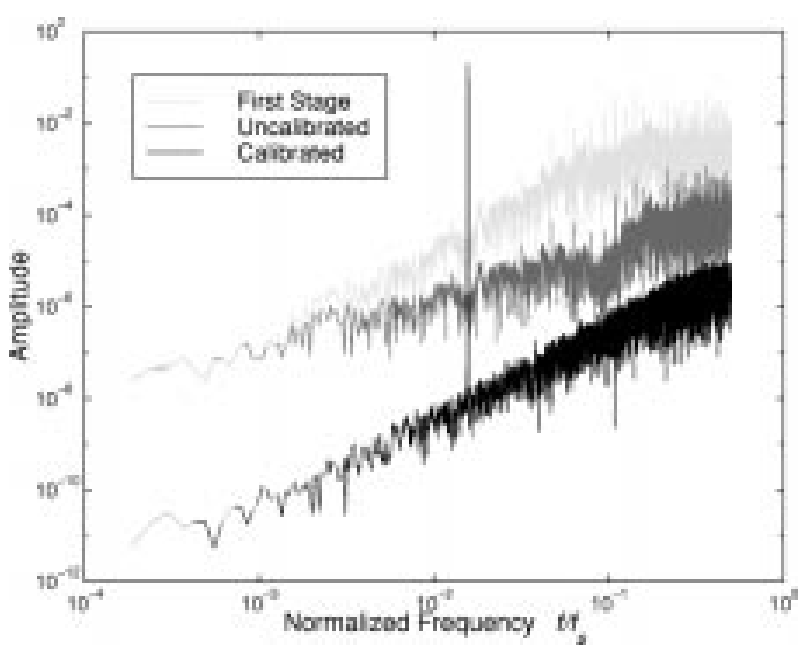

Fig. 7. Output amplitude spectrum in response to a sinusoidal input. Top: single-bit second-order $\Delta \Sigma$ modulator output $V_{0}$ from the first stage. Center: combined dual-quantization output $V$ from $\Delta \Sigma$ modulator and 16-stage radix 1.85 algorithmic A/D converter, with nominal, uncalibrated coefficients. Bottom: calibrated output $V^{c}$.

of $\Delta \Sigma$ modulation, and digital correction allows to recover the drastic loss in precision due to analog imperfections in the DAC [23].

As an example, assume that $Q_{m}$ is implemented as an algorithmic pipelined ADC, with a radix $\gamma$ less than two to warrant full digital correction capability in the presence of significant mismatch in the analog implementation [24]. "Ideal" correction of $V_{m}$ can be expressed as a linear combination of the bits obtained from the ADC [13]

$$
V_{m}^{c}=\sum_{k=0}^{K-1} \beta_{k} V_{m k}
$$

with bit coefficients $\beta_{k}, k=0, \cdots K-1$ determined by products of the ADC interstage gains, which can generally be different from $\gamma$. The linear expansion of $V_{m}$ with unknown $\beta_{k}$ coefficients can be directly included in the least-squares formulation for $E_{m}$ in (16), yielding

$$
\left|\mathcal{H}_{c} E_{m}\right|^{2} \approx\left|\sum_{i=0}^{m-1} F_{i} \mathcal{N}_{c} V_{i}+\sum_{k=0}^{K-1} \beta_{k} F_{m} \mathcal{N}_{c} V_{m k}\right|^{2} .
$$

The solution consists of minimizing (19) (in the time or frequency domain), while fixing one of the coefficients to eliminate one degree of freedom as before. The least-squares estimation is nonlinear in the parameters, since the coefficients in $F_{m}(z)$ and $\beta_{k}$ appear together in a product. A simple iterative means to the nonlinear optimization is to alternatingly solve for the $F_{i}(z)(i<m)$ and $\beta_{k}$ coefficients while fixing $F_{m}(z)$ (plus one other coefficient), and subsequently solving for $F_{i}(z)$ and $F_{m}(z)$ fixing the $\beta_{k}$ coefficients (plus one other). From nominal initial settings for $F_{m}(z)$, this procedure converges in between 5-20 iteration cycles, depending on calibration bandwidth (oversampling ratio), in our numerical experiments.

In the behavioral simulations, we consider a Leslie-Singh structure, $m=1$ in Fig. 3(a). A second-order delta-sigma modulator $l_{0}=2$, of the topology in Fig. 2(b), and an algo-

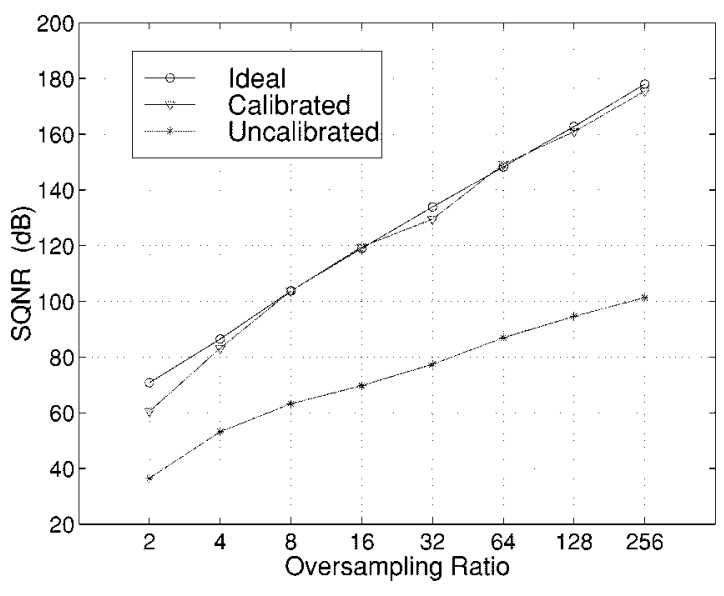

Fig. 8. SQNR as a function of oversampling ratio for the dual-quantization modulator, with ideal coefficients and calibrated coefficients obtained at maximum calibration bandwidth supported by the oversampling ratio.

rithmic A/D converter with $K=16$ stages and radix $\gamma=1.85$, are used. The analog parameter ranges correspond to typical switched-capacitor implementations: $a_{i}=0.5 \pm 0.01$ and $p_{i}=$ $0.9975 \pm 0.0025$.

The simulation results demonstrate significant improvements in SQNR achieved over the uncalibrated case, in which default values for the A/D coefficients and $\Delta \Sigma$ filters are assigned according to nominal values of the analog circuit parameters. As is evident from the modulation spectrum in response to a harmonic input in Fig. 7, the improvements result from a combination of eliminating dominant first-order quantization noise from the first stage, and linearizing the pipelined A/D multibit quantizer. By forcing a flat spectrum for $E_{1}$ in (16), harmonic distortion present in the $V_{1}$ output, due to strong correlations between $U_{1}$ and $U_{0}$, is largely eliminated.

The dependence of SQNR on the oversampling ratio is shown in Fig. 8. The blind calibration technique achieves near-perfect correction at higher oversampling ratios where the off-line method saturates. The increasing success of the blind method at higher oversampling ratios reflects the larger portion of the spectrum covered by the calibration band.

The astronomical figures of SQNR are shown only to illustrate the correction power of the calibration procedure, which clearly goes beyond practical limits that are physically attainable with noise levels present in typical analog circuits. To verify the robustness of the above calibration techniques under more general conditions, we have conducted simulations including additive noise in each of the stages (both integrators and quantizers), showing no significant degradations in SNR performance obtained from noisy calibration compared to that of the ideal coefficients under identical noisy conditions.

\section{DisCUSSION}

\section{A. Blind versus Reference-Based Calibration}

The robust performance of the blind on-line calibration technique under fairly general conditions may seem to suggest that there is no need for another scheme. There are two important issues that factor in the comparison with reference-based calibration schemes. 
1) The formulation of blind calibration restricts the input to be bandlimited. This could, in principle, be satisfied by means of an anti-alias front-end filter, although such a filter is not strictly needed for an oversampling data converter and may aversely affect the overall SNDR performance. Without the low-pass front-end, some signal may leak through in the calibration band. The effect of this leakage is minimal, as long as it remains below the quantization noise floor, which is elevated in the calibration band as a result of noise shaping.

2) The absence of an absolute signal reference in blind calibration leaves the signal gain undefined. This becomes an issue in certain applications where absolute accuracy is crucial. It is particularly important for time-interleaved or parallel data converters, where random gain variations introduce cyclic or fixed pattern noise. The pseudo on-line reference-based calibration technique in Section IV-B would be best suited in this case.

Similar concerns apply to on-line reference-based schemes in Section IV-C as to the blind scheme: the implicit assumption is that the input signal and quantization noise of intermediate stages do not correlate with the reference, and the signal gain of the corrected output may be in error because the reference gain is generally different from the signal gain at the summing node where both signals are (capacitively) combined.

The analog hardware requirements for all reference-based methods are similar, including a precise (pseudo-random or other two-level) reference, and additive (i.e., capacitive) access to the reference injection node(s). This overhead in hardware is very modest, and the main issue in the implementation (by construction) is the complexity of digital correction and adaptive calibration.

\section{B. Hardware Complexity}

The digital correction and adaptation algorithms could readily be implemented in real time on a host or supporting digital processor, incurring no extra hardware, but a significant processor load. Some simplifications below make it practical to integrate these functions along with the analog circuitry at a minimal cost in complexity of implementation.

The digital correction by itself requires a bank of FIR filters $F_{i}(z)$, as shown in the digital part of Fig. 3. The number of taps depends on the number of stages and the order of each submodulator, as given by (13), but is relatively small (ten and six total coefficients in the above off-line and blind on-line calibration case studies, respectively, including one coefficient in the blind on-line case that can be set to unity). The coarse (1-bit or K-bit) quantization in the $v_{i}[k]$ outputs also reduces the size (number of bits) of the FIR multipliers.

Adaptive calibration of the FIR coefficients $f_{i j}$, where $F_{i}(z)=\sum_{j} f_{i j} z^{-j}$, is conveniently implemented using the delta rule or its variants. This applies to both blind and reference-based methods. For instance, gradient descent of the off-line metric (15) leads to the update rule

$$
\begin{aligned}
\Delta f_{i j}[n] & =f_{i j}[n+1]-f_{i j}[n] \\
& =-\eta \mathcal{L}_{c} v_{i}[n-j]\left(\mathcal{L}_{c} v[n]-\mathcal{L}_{c} u^{\mathrm{cal}}[n-L]\right)
\end{aligned}
$$

of which a simplified implementation, with identical steady state solution (over an infinite horizon), is constructed by rearranging contributions over time

$$
\Delta f_{i j}[n]=-\eta v_{i}[n-j] \mathcal{L}_{c} \mathcal{L}_{c}^{*}\left(v[n]-u^{\mathrm{cal}}[n-L]\right)
$$

thus avoiding the need to decimate each of the quantized outputs $v_{i}$ individually, but the decimated error instead. (This comes at the expense of a need for extra delays in $v_{i}$ to get around the noncausality of the conjugated decimation filter, i.e., time-reversed impulse response.) Multiplication reduces to a single-bit exclusive-or (XOR) by adopting a pilot-rule version of (21)

$$
\begin{aligned}
\Delta f_{i j}[n]=-\eta \operatorname{sign}( & \left.v_{i}[n-j]\right) \\
& \cdot \operatorname{sign}\left(\mathcal{L}_{c} \mathcal{L}_{c}^{*}\left(v[n]-u^{\operatorname{cal}}[n-L]\right)\right)
\end{aligned}
$$

implemented using one up/down counter per coefficient $f_{i j}$ for $\eta \equiv 1$. Adaptive schemes are available to dynamically adjust the "learning rate" constant $\eta$ for improved rate of convergence [25].

The same simplifying transformations can be applied to implement on-line reference-based and blind adaptive calibration schemes, with all filtering in the gradient-based LMS formulation referred to the output (residue), and pilot versions implemented in the form of counters. A particular architecture for LMS-based digital adaptation is included in Part II of the paper.

\section{CONCLUSION}

We have presented a simple calibration technique for adaptive digital correction of multiple quantization delta-sigma modulators. The calibration effectively removes quantization noise that leaks through at the output due to analog imperfections in the implementation, by means of adjusting the coefficients of the differentiation filters to the realized analog transfer functions of the modulation stages. Our approach also allows the correction of spectral distortion and gain mismatch in the signal path due to a nonideal signal transfer function in the modulators, and extends to adaptive correction of static nonlinearity in Nyquist-rate converters.

Under the mild assumption of a bandlimited input signal, we have demonstrated that on-line digital calibration is possible using no more information than direct observations of the digital outputs being calibrated, with no need to apply a signal reference or interrupting the data conversion process. The procedure is widely applicable to multistage designs, and was verified with behavioral simulations on a Leslie-Singh dual-stage multibit oversampled A/D converter with a pipelined algorithmic A/D quantizer, with SQNR improvements beyond physical accuracy limits in analog circuit implementations.

An alternative on-line calibration technique, based on injection of known random signals at select points in the analog architecture, is presented in the companion paper [1]. Further practical considerations, as well as experimental results from silicon, are also included there.

\section{REFERENCES}

[1] P. Kiss, J. Silva, A. Wiesbauer, T. Sun, U.-K. Moon, J. T. Stonick, and G. C. Temes, "Adaptive digital correction of analog errors in MASH ADCs-Part II: Correction using test-signal injection," IEEE Trans. Circuits Syst. II, pp. 629-638, this issue. 
[2] J. C. Candy and G. C. Temes, "Oversampled methods for A/D and D/A conversion," in Oversampled Delta-Sigma Data Converters. Piscataway, NJ: IEEE Press, 1992, pp. 1-29.

[3] T. Hayashi, Y. Inabe, K. Uchimura, and T. Kimura, "A multistage deltasigma modulator without double integration loop," ISSCC Tech. Dig. Pap., vol. 39, pp. 182-183, 1986.

[4] K. Uchimura, T. Hayashi, T. Kimura, and A. Iwata, "Oversampled A-to-D and D-to-A converters with multistage noise shaping modulators," IEEE Trans. Acoust., Speech, Signal Processing, vol. 36, pp. 1899-1905, Dec. 1988.

[5] T. C. Leslie and B. Singh, "An improved sigma-delta modulator architecture," in Proc. ISCAS '90, 1990, pp. 372-375.

[6] _ - "Sigma-delta modulators with multi-bit quantizing elements and single-bit feedback," Proc. Inst. Elect. Eng. G, vol. 139, no. 3, pp. 356-362, 1992.

[7] D. B. Ribner, "A comparison of modulator networks for high-order oversampling sigma-delta analog-to-digital conversion," IEEE Trans. Circuits Syst., vol. 38, pp. 145-159, 1991.

[8] S. Au and B. H. Leung, "A 1.95-V, 0.34-mW, 12-b sigma-delta modulator stabilized by local feedback loops," IEEE J. Solid-State Circuits, vol. 32, pp. 321-328, Mar. 1997.

[9] G. Cauwenberghs and G. C. Temes, "Adaptive calibration of multiple quantization oversampled A/D converters," in Proc. IEEE Int. Symp. Circuits and Systems (ISCAS'96), vol. I, Atlanta, GA, 1996, pp. $512-515$.

[10] Y. Yang, R. Schreier, G. C. Temes, and S. Kiaei, "On-line adaptive digital correction of dual-quantization delta-sigma modulators," Electron. Lett., vol. 28, no. 16, pp. 1511-1513, 1992.

[11] S. Abdennadher, S. Kiaei, G. C. Temes, and R. Schreier, "Adaptive selfcalibrating delta-sigma modulators," Electron. Lett., vol. 28, no. 14, pp. 1288-1289, 1992.

[12] A. Wiesbauer and G. C. Temes, "Adaptive digital compensation of analog circuit imperfections for cascaded $\Delta \Sigma$ modulators," in Proc. 30th Asilomar Conf. Signals, Systems and Computers, Pacific Grove, CA, Nov. 3-6, 1996.

[13] G. Cauwenberghs, "Blind on-line digital calibration of multi-stage nyquist-rate and oversampled A/D converters," in Proc. IEEE Int. Symp. Circuits and Systems (ISCAS'98), vol. I, Monterey, CA, 1998, pp. 401-404.

[14] T. Sun, A. Wiesbauer, and G. C. Temes, "Adaptive compensation of analog circuit imperfections for cascaded delta-sigma ADCs," in Proc. IEEE Int. Symp. Circuits and Systems (ISCAS'98), vol. I, Monterey, CA, 1998, pp. 405-407.

[15] R. Gregorian and G. C. Temes, Analog MOS Integrated Circuits for Signal Processing. New York: Wiley, 1986.

[16] J. C. Candy, "The use of double integration in sigma delta modulation," IEEE Trans. Commun., vol. COM-33, pp. 249-258, Mar. 1985.

[17] B. E. Boser and B. Wooley, "The design of sigma-delta modulation analog-to-digital converters," IEEE J. Solid-State Circuits, vol. SC-23, pp. 1298-1308, June 1988.

[18] J. Steensgaard-Madsen, High-Performance Data Converters, Dept. of Info. Technol., Tech. Univ. of Denmark, Denmark, Mar. 8, 1999.

[19] A. Petraglia and S. K. Mitra, "Analysis of mismatch effects among A/D converters in a time-interleaved waveform digitizer," IEEE Trans. Instrum. Meas., vol. 40, pp. 831-835, Mar. 1991.

[20] A. Petraglia and S. K. Mitra, "High-speed A/D conversion incorporating a QMF bank," IEEE Trans. Instrum. Meas., vol. 41, pp. 427-431, Mar. 1992.

[21] I. Galton, "Analysis of quantization noise in $\Delta \Sigma$ modulation and its application to parallel $\Delta \Sigma$ modulation," Ph.D. dissertation, California Inst. of Technol., Pasadena, CA, 1992.

[22] R. Khoini-Poorfard, L. B. Lim, and D. A. Johns, "Time-interleaved oversampling A/D converters: Theory and practice," IEEE Trans. Circuits Syst. II, vol. 44, pp. 634-645, Aug. 1997.

[23] M. Sarhang-Nejad and G. C. Temes, "A high-resolution multibit $\Delta \Sigma$ ADC with digital correction and relaxed amplifier requirements," IEEE J. Solid-State Circuits, vol. 28, pp. 648-660, June 1993.
[24] A. N. Karanicolas, H.-S. Lee, and K. L. Bacrania, "A 15-b 1-Msample/s digitally self-calibrated pipeline ADC," IEEE J. Solid-State Circuits, vol. 28, pp. 1207-1215, Dec. 1993.

[25] G. Cauwenberghs and M. A. Bayoumi, Eds., Learning on Silicon. Norwell, MA: Kluwer, 1999.

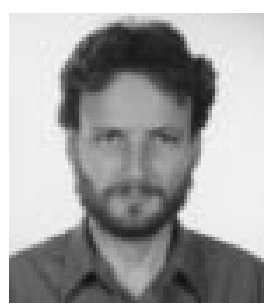

Gert Cauwenberghs (S'89-M'94) received the Engineer's degree in Applied Physics from the University of Brussels, Belgium, in 1988, and the M.S. and Ph.D. degrees in electrical engineering from the California Institute of Technology, Pasadena, CA, in 1989 and 1994.

In 1994, he joined Johns Hopkins University, Baltimore, MD, where he is now Associate Professor of Electrical and Computer Engineering. During 1998-1999, he was on sabbatical as Visiting Professor of Brain and Cognitive Science at the Center for Computational and Biological Learning, Massachusetts Institute of Technology, Cambridge, MA, and at the Center for Adaptive Systems, Boston University, Boston, MA. He recently co-edited the book Learning on Silicon (Norwell, MA: Kluwer, 1999). His research covers VLSI circuits, systems and algorithms for parallel signal processing, adaptive neural computation, and low-power coding and instrumentation.

Dr. Cauwenberghs is an Associate Editor of the IEEE TRANSACTIONS ON Circuits AND Systems II: ANALOG AND Digital Signal Processing, and Vice Chair of the IEEE Circuits and Systems Society Technical Committee on Analog Signal Processing. He has organized special sessions at conferences and special issue journals on learning, adaptation, and memory. He was Francqui Fellow of the Belgian American Educational Foundation in 1988, and received the National Science Foundation Career Award in 1997, the Office of Naval Research Young Investigator Award in 1999, and the Presidential Early Career Award for Scientists and Engineers (Pecase) in 2000.

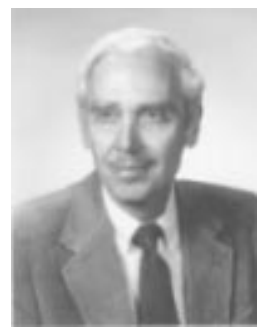

Gabor C. Temes (SM'66-F'73-LF'98) received the undergraduate degree from the Technical University and Eötvös University, Budapest, Hungary, from 1948 to 1956, and the Ph.D. degree in electrical engineering from the University of Ottawa, Canada, in 1961. He received an honorary doctorate from the Technical University of Budapest in 1991.

$\mathrm{He}$ held academic positions at the Technical University of Budapest, Stanford University, and at UCLA, and worked in industry at Northern Electric R\&D Laboratories (now Bell-Northern Research) and Ampex Corporation. He is now a Professor in the Department of Electrical and Computer Engineering, Oregon State University (OSU), Corvallis, OR. He served as Department Head at both UCLA and OSU. He is co-editor and co-author of Modern Filter Theory and Design (New York: Wiley, 1973), co-author of Introduction to Circuit Synthesis and Design (New York: McGraw-Hill, 1977), co-author of Analog MOS Integrated Circuits for Signal Processing (New York: Wiley, 1986), and co-editor and co-author of both Oversampling Delta-Sigma Data Converters (Piscataway, NJ: IEEE Press, 1992) and Delta-Sigma Data Converters (Piscataway, NJ: IEEE Press, 1997). He is a contributor to several other edited volumes, and has published approximately 300 papers in engineering journals and conference proceedings. His recent research has dealt with CMOS analog integrated circuits, as well as data converters and integrated sensor interfaces.

Dr. Temes was an Associate Editor of the Journal of the Franklin Institute, Editor of the IEEE TRANSACTIONS ON CiRCUIT THEORY, and Vice President of the IEEE Circuits and Systems Society (CAS). He was co-recipient of the CAS Darlington Award in 1968 and 1981, and winner of the Centennial Medal of the IEEE in 1984. He received the Andrew Chi Prize Award of the IEEE Instrumentation and Measurement Society in 1985, the Education Award of the IEEE CAS Society in 1987, the CAS Technical Achievement Award in 1989, the IEEE Graduate Teaching Award in 1998, and the IEEE Millennium Medal and the IEEE CAS Golden Jubilee Medal in 2000. 